\title{
Segue Between Favorable and Unfavorable Solvation
}

\author{
Lutz Maibaum and David Chandler \\ Department of Chemistry, University of California, Berkeley, California 94720
}

(Dated: May 21, 2007)

\begin{abstract}
Solvation of small and large clusters are studied by simulation, considering a range of solvent-solute attractive energy strengths. Over a wide range of conditions, both for solvation in the Lennard-Jones liquid and in the SPC model of water, it is shown that the mean solvent density varies linearly with changes in solvent-solute adhesion or attractive energy strength. This behavior is understood from the perspective of Weeks' theory of solvation [Ann. Rev. Phys. Chem. 2002, 53, 533] and supports theories based upon that perspective.
\end{abstract}

\section{INTRODUCTION}

Some solute species dissolve in a liquid solvent over a large range of conditions. Others demix or phase separate from the liquid solvent even at relatively low solute concentrations. This paper focuses on mesoscopic and microscopic manifestations of these macroscopic phenomena. Our motivation is to understand the range of solvation behaviors that are possible in aqueous solutions when hydrophobic species are altered to hydrophilic and vice versa. We demonstrate that various physical quantities vary smoothly under such alterations, metaphorically akin to a segue that smoothly bridges two distinct musical movements. While the motivation concerns this specific class of solutions, the physical principles involved are relatively general.

The basic theory has been formulated by Weeks [1]. One first considers the effect of the 
solute in excluding a region of space from the solute. The average effect is described by the response of the solvent to a so-called unbalancing potential. Unbalancing arises because the excluded volume presents a region where solvent molecules lose adhesive forces. In a homogeneous fluid, these adhesive forces tend to be in balance, often allowing their effects to be described as a uniform field or chemical potential shift $[2,3]$. Introducing a large enough excluded volume, however, destroys this homogeneity, and the response can be very large, particularly so if the liquid exists near coexistence with its vapor. Indeed, in the macroscopic limit, the average liquid density adjacent to excluded regions bounded by a plane is $\beta p$, where $p$ is the pressure, and $\beta^{-1}=k_{\mathrm{B}} T$ is temperature times Boltzmann's constant [4]. In this limit, therefore, the mean liquid density at the surface is a bulk property. It exhibits a phase transition reflecting the bulk liquid-vapor phase transition. Weeks' unbalancing potential captures the effects of this transition in a mean molecular field approximation.

A typical solute does not exclude liquid from a macroscopic volume. Nevertheless, the unbalancing potential can be substantial and the response to it significant, though not singular, provided the solvent-exposed surface area of the solute has low enough curvature and large enough size. Weeks' expression for the unbalancing potential shows that its strength is roughly proportional to the surface area, $A$. Thus, to linear order in the response,

$$
\langle\rho(\mathbf{r})\rangle_{0} \approx a(\mathbf{r})-A b(\mathbf{r}) .
$$

Here, $\rho(\mathbf{r})$ denotes the solvent density field at position $\mathbf{r}$, which we take to be outside the excluded volume, and the pointed brackets indicate equilibrium ensemble average, with the subscript 0 implying that only excluded volume forces act between solute and solvent. The function $a(\mathbf{r})$ and $b(\mathbf{r})$ are properties of the solvent and the solute position and shape; $a(\mathbf{r})$ gives the response in the absence of unbalancing forces; $A b(\mathbf{r})$ is significant in size only when the liquid solvent is close to phase coexistence with its vapor and $A$ is comparable to 
or larger than the surface area of the critical nucleus for the phase transition; for positions $\mathbf{r}$ that are beyond a bulk correlation length of the solvent, $a(\mathbf{r})$ approaches the bulk liquid density, and $b(\mathbf{r})$ approaches zero.

Fluctuations around the average and the effects of forces beyond those of excluded volume are captured in the second step of Weeks' treatment. In this step, one notes that solvent density fluctuations in a homogeneous liquid obey Gaussian statistics [5-7], and assumes this statistics is more generally valid for all small length scale fluctuations in a liquid, even in the presence of inhomogeneities. Therefore, if the solute attracts the solvent molecules with a potential strength proportional to $\lambda$, the effect of the attraction on the mean solvent density is linear in $\lambda$, i.e., $\langle\rho(\mathbf{r})\rangle_{\lambda} \approx\langle\rho(\mathbf{r})\rangle_{0}+\lambda S(\mathbf{r})$, where $S(\mathbf{r})$ is the net or integrated response of the solvent density field at $\mathbf{r}$ to the attractions from the solute. If these attractions emanate from interaction sites in the solute and are finite ranged, then $S(\mathbf{r})$ will be approximately linear in $A$, leading to

$$
\langle\rho(\mathbf{r})\rangle_{\lambda} \approx\langle\rho(\mathbf{r})\rangle_{0}+\lambda A s(\mathbf{r}),
$$

where $s(\mathbf{r})$ is a property of the solvent and the functional form of the attractive interaction. Finally, as both $b(\mathbf{r})$ and $s(\mathbf{r})$ describe responses of the solvent near the solute and per unit exposed area of the solute, one may expect that integrated or coarse-grained effects of the two are approximately proportional. That is to say, $\bar{b}(\mathbf{r}) \approx \lambda^{*} \bar{s}(\mathbf{r})$, where the over-bar indicates an integral or coarse-graining over some specified microscopic length scale. The value of the proportionality constant, $\lambda^{*}$, depends upon the specific solvent, the specific solute-solvent interactions, and the specific coarse-graining perscription. Adopting this proportionality gives

$$
\langle\bar{\rho}(\mathbf{r})\rangle_{\lambda} \approx \bar{a}(\mathbf{r})+\left(\lambda-\lambda^{*}\right) A \bar{s}(\mathbf{r}) .
$$

Equations (1), (2) and (3) are not generally true, as they do not properly account for 
saturation in either the limit of very large $A$ or the limit of very large $\lambda$. The point of this paper, however, is that these equations are good approximations in a variety of physically pertinent circumstances. We demonstrate their accuracy in the next section by presenting results of computer simulation calculations of solvent density fields and solvation free energies for solute clusters of various sizes and solvent-solute attractive energy strengths, considering both the Lennard-Jones liquid solvent and water solvent. The results indicate that variations from favorable to unfavorable solvation, i.e., the changes in solvation in passing from large $\lambda$ to small $\lambda$, occur smoothly and in ways that are easy to anticipate. Further, the results provide confidence in theories built from Weeks' perspective. These implications are discussed in Section III.

\section{SIMULATION RESULTS}

To elucidate the response of a liquid to the presence of a solute of general shape and interaction strength, we consider a series of models for both solutes and solvent of increasing complexity.

\section{A. Spherical solutes in Lennard-Jones solvent}

First we consider the case of a spherical solute immersed in the Lennard-Jones solvent, i.e., a fluid of particles that interact through the pair potential

$$
w_{\mathrm{LJ}}(r)=4 \varepsilon\left[\left(\frac{\sigma}{r}\right)^{12}-\left(\frac{\sigma}{r}\right)^{6}\right]
$$

In this equation $r$ denotes the distance between two solvent particles, and $\varepsilon$ and $\sigma$ define the energy and length scales of the simulation. The solvent-solvent potentials are shifted so as to be continuous and zero beyond the cut-off distance $r_{\mathrm{c}}=2.5 \sigma$. The reduced thermodynamic 
state conditions for the solvent are $k_{\mathrm{B}} T / \varepsilon=0.85, p \sigma^{3} / \varepsilon=0.022$ and $\rho \sigma^{3}=0.7$, where $\rho$ is the bulk density. These conditions place this Lennard-Jones liquid with truncated and shifted interaction potentials at or very close to liquid-vapor coexistence $[8,9]$.

The solute is a sphere of radius $R$, and its interaction with the solvent is that of a sphere consisting of a Lennard-Jones material with homogenous density equal to that of the solvent. A solvent particle at distance $r$ from the solute center has the potential energy[10]

$$
\Phi(r)=\pi \varepsilon \rho \sigma^{3}\left[\frac{4}{5} \sigma^{9}\left(\frac{1}{8 r r_{+}^{8}}-\frac{1}{9 r_{+}^{9}}-\frac{1}{8 r r_{-}^{8}}+\frac{1}{9 r_{-}^{9}}\right)-2 \sigma^{3}\left(\frac{1}{2 r r_{+}^{2}}-\frac{1}{3 r_{+}^{3}}-\frac{1}{2 r r_{-}^{2}}+\frac{1}{3 r_{-}^{3}}\right)\right],
$$

where $r_{ \pm}=r \pm R$, if $r \geq R$, and $\phi(r)=\infty$ otherwise. This potential is minimal at a distance $r_{\min }$, and its minimum value $\Phi_{\min }=\Phi\left(r_{\min }\right)$ depends on the solute size $R$.

To systematically investigate the role of attractive interactions, we employ the scheme introduced by Weeks, Chandler and Andersen (WCA) to partition the energy (5) into the short-ranged repulsive and the long-ranged attractive part [3],

$$
\Phi_{\lambda}(r)=\Phi_{0}(r)+\lambda \Phi_{1}(r)
$$

where

$$
\Phi_{0}(r)=\left\{\begin{array}{cl}
\Phi(r)-\Phi\left(r_{\text {min }}\right) & \text { if } r \leq r_{\text {min }} \\
0 & \text { if } r>r_{\text {min }}
\end{array}\right.
$$

and

$$
\Phi_{1}(r)=\left\{\begin{array}{cl}
\Phi\left(r_{\min }\right) & \text { if } r \leq r_{\min } \\
\Phi(r) & \text { if } r>r_{\min } .
\end{array}\right.
$$

The parameter $\lambda$ controls the strength of the attractive solute-solvent interactions.

We performed computer simulations using the Monte Carlo method at fixed pressure and temperature for different values of the solute size $R$ and interaction strength $\lambda$. The number $N$ of solvent particles ranged from 864 to 8192 depending the size of the solute. 
The solute-solvent interaction (6) was truncated and shifted at a distance $r=R+2.5 \sigma$. We calculate the average reduced solvent density at position $\boldsymbol{r}$,

$$
g(\boldsymbol{r})=\langle\rho(\boldsymbol{r})\rangle / \rho
$$

in an ensemble where the solute position is fixed at $\boldsymbol{r}=0$. Due to the isotropic nature of the solute this density depends only on the distance $r=|\boldsymbol{r}|$ from the solute center.

We find that the reduced density $g(r)$ has generally a well-defined first peak corresponding to the first solvation shell of the solute. The height of this peak, denoted $g_{\max }$, is shown in the top panel of Fig. 1 for different values of $R$ and $\lambda$. This graph encompasses many of the important effects of our understanding of solvophobic and solvophilic solvation.

First, for a fixed value of $\lambda$ less than 0.9 , the solvent density near the solute decreases with increasing solute radius. It has been observed previously that the contact density near a hard sphere solute decreases from values above the bulk liquid density at small solute sizes to the equilibrium vapor density at large solute sizes, both for the Lennard--Jones solvent [11] and water [10]. The creation of a liquid-vapor interface near large enough solutes is the basis of our understanding of the hydrophobic effect [12]. Our simulations show that this behavior persists for solutes with weak but finite attraction strengths $\lambda$.

Second, for any given solute size $R$, the density near the solute increases approximately linearly with the attraction strength $\lambda$. This smooth response of the density to an attractive field over a wide range of interactions strengths shows that the solvent properties do not change significantly as $\lambda$ increases. In particular it is consistent with Gaussian density fluctuations for all attraction strengths.

Third, it is apparent that the slope $\partial g_{\max } / \partial \lambda$, while independend of $\lambda$, depends on the radius $R$ of the solute. In particular, there is a value $\lambda^{*} \approx 0.9$ of the interaction strength where the mean density itself seems to be independent of the solute size. A similar result 
is suggested by Ashbaugh and Paulaitis for the density near methane clusters in water [13]. This behavior can be easily understood within the framework introduced in Section I. The numerical value of $\lambda$ by itself is not an accurate parameter to describe the strength of the solute-solvent interactions. For the special case of the homogeneous Lennard--Jones solute, a much more reliable indicator is the magnitude of the minimum in the potential energy, $\lambda\left|\Phi_{\min }(R)\right|$. The bottom panel of Fig. 1 shows that the density response is the same for all solute sizes if the control parameter is chosen to be the actual magnitude of the solute-solvent attractions.

Our results for this simple model solute serve as a useful illustration for the theory outlined in Section I. We now turn to more complicated solutes, where the lack of isotropy demands the analysis of more complicated density distribution functions. While these complications can obscur the underlying physics, we will see that the basic picture of solvation presented here remains correct.

\section{B. Cluster solutes in Lennard-Jones solvent}

We consider clusters of $n$ particles solvated in the same Lennard-Jones fluid as before. The clusters are the model solutes of Ashbaugh and Paulaitis [13]. Space filling views are shown in Figure 2. The clusters are composed of $n=1,13,57,135$, and 305 hexagonally

close packed particles with nearest neighbor distance $d=2^{1 / 6} \sigma$. The solute interacts with the solvent molecules with the potential energy

$$
W_{\lambda}=\sum_{i=1}^{N} \sum_{\alpha=1}^{n} w_{\lambda}\left(\left|\boldsymbol{r}_{\alpha}-\boldsymbol{R}_{i}\right|\right)
$$


where $\boldsymbol{r}_{\alpha}$ is the position of the $\alpha$ th cluster particle, $\boldsymbol{R}_{i}$ is the position of the $i$ th solvent particle, and

$$
w_{\lambda}(r)=w_{0}(r)+\lambda w_{1}(r)
$$

Here, $w_{0}(r)$ and $w_{1}(r)$ are, respectively, the repulsive branch and the attractive branch of the Lennard-Jones potential [3],

$$
w_{0}(r)=\left\{\begin{array}{cl}
4 \varepsilon_{\mathrm{s}}\left[\left(\sigma_{\mathrm{s}} / r\right)^{12}-\left(\sigma_{\mathrm{s}} / r\right)^{6}+\frac{1}{4}\right] & \text { if } r \leq 2^{1 / 6} \sigma_{\mathrm{s}} \\
0 & \text { if } r>2^{1 / 6} \sigma_{\mathrm{s}}
\end{array}\right.
$$

and

$$
w_{1}(r)=\left\{\begin{array}{cc}
-\varepsilon_{\mathrm{s}} & \text { if } r \leq 2^{1 / 6} \sigma_{\mathrm{s}} \\
4 \varepsilon_{\mathrm{s}}\left[\left(\sigma_{\mathrm{s}} / r\right)^{12}-\left(\sigma_{\mathrm{s}} / r\right)^{6}\right] & \text { if } r>2^{1 / 6} \sigma_{\mathrm{s}}
\end{array} .\right.
$$

We choose $\sigma_{\mathrm{s}}=\sigma$ and $\varepsilon_{\mathrm{s}}=\varepsilon$ so that the interaction between a cluster particle and a solvent particle is the same as that between two solvent particles at $\lambda=1$.

The $n=1$ solute is spherically symmetric, and the mean solvent density near it is described by the isotropic radial distribution function $g(r)$ as before. For the clusters with $n>1$, however, specification of the mean solvent density requires many more variables than $r$, and it is natural to consider projections of the mean density. One such projection is the so-called proximal distribution $g_{\text {prox }}(r)$, defined as the reduced density averaged over the surface $[13,14]$

$$
\left\{\boldsymbol{r}^{\prime} \in \mathbb{R}^{3}: r_{\text {prox }}\left(\boldsymbol{r}^{\prime}\right)=r\right\}
$$

where

$$
r_{\text {prox }}(\boldsymbol{r})=\min \left\{\left|\boldsymbol{r}-\boldsymbol{r}_{\alpha}\right|: \alpha=1, \ldots, n\right\}
$$

is the distance from a point $\boldsymbol{r}$ to the nearest cluster particle. While explicitly a function of only one length, $r$, it is implicitly a multi-point distribution function [15], except in the case $n=1$. For that special case $g_{\text {prox }}(r)$ is the standard radial distribution function $g(r)$. 
Figure 3 illustrates the behavior of the proximal distribution function for the model solutes in the Lennard-Jones solvent. Ashbaugh and Paulaitis proposed the height $g_{\max }$ of the first peak in $g_{\text {prox }}(r)$ as a meaningful measure of the solvent density near arbitrary solutes. In the top panel we show that the behavior of this observable is qualitatively the same as that shown in Fig. 1 for isotropic solutes. The response of the mean solvent density to changes in solute-solvent attraction is proportional to that change. This result is as expected according to Eq. (2). Another important point is that this proportionality leads to a coincidence of curves at $\lambda=\lambda^{*}$, where in this case $\lambda^{*} \approx 0.5$. The existence of a coincident value of $\lambda$ is anticipated in Eq. (3).

Ashbaugh and Paulaitis found in their study of methane clusters solvated in water that at $\lambda^{*}$ not only the heights of the first peak in $g_{\text {prox }}(r)$ coincide for different cluster sizes, but that the proximal distribution functions themselves are very similar. The bottom panel of Fig. 3 establishes a similar result for our model system. From this behavior, Ashbaugh and Paulaitis conclude that the solvation environment of a monomeric methane particle is essentially the same as that of methane confined to a cluster of arbitrary size. Fig. 4 shows that this conclusion is not justified. The contour lines of constant density do not coincide with the surfaces of constant proximal distance for the $n=135$ cluster. In particular, the crevices of the solute, where the attraction strength is large, contain a much higher solvent density than the proximal distribution function suggests. No such behavior exists for the monomeric solute, where the density profile is isotropic.

As the strength of the attactive solute-solvent interaction increases, the clusters lose their hydrophobic character until eventually they become hydrophilic. To quantify this process we calculate the solvation free energy, or excess chemical potential, as a function of $\lambda$, which 
is given by

$$
\Delta \mu_{\lambda}=\Delta \mu_{0}+\int_{0}^{\lambda} \mathrm{d} \lambda^{\prime}\left\langle W_{1}-W_{0}\right\rangle_{\lambda^{\prime}}
$$

where $\langle\ldots\rangle_{\lambda}$ indicates ensemble average with solute-solvent attractive force strength $\lambda$.

Solvation free energies due to excluded volume are approximately proportional to the excluded volume for small solutes. Further, at large excluded volumes with small surface curvature and no attractive solvent-solute forces, solvent density is depleted near the solute. Therefore, for the solutes we have considered, $\Delta \mu_{0}$ can be well approximated by the solvation free energy for a hard sphere with the same excluded volume as the solute clusters [11]. Here we define the excluded volume as those spatial regions where the solute-induced field is greater than the thermal energy $k_{\mathrm{B}} T$.

The result of this calculation is shown in Fig. 5. Over the range of interaction strengths considered, the solvation free energies change from strongly positive to negative. In other words, at large values of $\lambda$ the clusters are not solvophobic. This transition occurs smoothly and regularly. The shape of these curves is understood, because the attractive energy is approximately proportional to $\lambda$ times an integral over $\langle\rho(\boldsymbol{r})\rangle_{\lambda}$, and the latter is a linear function of $\lambda$, as noted in Eq. (2).

\section{Cluster solutes in SPC water}

In another series of calculations, the solvent is the SPC model for liquid water [16], and the solutes are clusters of methane molecules in the united atom approximation of Ref. 17. As in Ref. 13 these clusters are hexagonally close-packed with a particle spacing of $d=4.19 \AA$. The methane molecules interact with the water oxygens through a LennardJones potential with $\sigma=3.448 \AA$ and $\varepsilon=0.895 \mathrm{~kJ} / \mathrm{mol}$, which was truncated and shifted 
at a distance of $9 \AA$. As before we use the WCA partition of the interaction potential to obtain control over the strength of the attractions, given by the parameter $\lambda$. Electrostatic interactions were calculated using Ewald summation. Molecular dynamics simulations were performed using the LAMMPS program [18, 19] at temperature $T=298 \mathrm{~K}$ and a box size chosen to give the correct bulk density [13].

As noted above, Ashbaugh and Paulaitis [13] found that the proximal solvent density distribution functions are very similar for different cluster sizes at the specific attraction strength $\lambda^{*} \approx 1$, from which they concluded that the solvent density near a cluster of solute particles is basically the same as that near a single solute particle. In Fig. 6 we show the reduced water density near the $n=135$ solute with an interaction strength $\lambda=\lambda^{*}$. For this specific system the solvent density is indeed approximately constant along contours of constant proximal distance, so that

$$
\langle\rho(\boldsymbol{r})\rangle \approx \rho g_{\mathrm{prox}}\left(r_{\mathrm{prox}}(\boldsymbol{r})\right)
$$

is a rather good approximation for the range of parameters considered. However, this agreement is not exact, as deviations are visible in the crevices. In these regions adjacent to the cluster, the solvent interacts with many solute particles, and the attractive potential well reaches values as deep as $-5 \mathrm{~kJ} / \mathrm{mol}$. This value is to be compared with the average energy of a water molecule in the bulk fluid, which is approximately $40 \mathrm{~kJ} / \mathrm{mol}$ [20]. Thus, the cluster crevices are approaching hydrophilic character and can compensate for a fraction of the average water-water interaction energy.

Fig. 7 shows the solvation free energy of the methane clusters in water, calculated using (16) as before. Again the clusters show a smooth transition from hydrophobic solvation at small values of $\lambda$ to hydrophilic solvation at large attraction strengths. For this particular system, the crossover between these two regimes occurs at values of $\lambda$ greater than $\lambda^{*}$. 


\section{DISCUSSION}

The results of the previous section show that the role of solvent-solute attractions on solvation is significant but simple. In particular, while fluctuations of solvent density are affected by the shape and size of the solute and the thermodynamic state of the solvent, the mean solvent density changes only linearly with changes in the strength of adhesion between solute and solvent. If, for example, a solute is small, the liquid solvent will be constrained to be outside the solute's excluded volume but otherwise behave as if it were bulk. This conception is precisely the physics that underlies the Pratt-Chandler theory of hydrophobicity $[7,21]$ and its contemporary variant [5]. On the other hand, if the solute's girth is such to exclude a volume with large low-curvature surface area, fluctuations of the solvent are changed to reflect the formation of an interface $[1,12,22]$. To the extent there is no solvent-solute adhesion, the interface is essentially the same as that between liquid and vapor [23]. The role of attractions between the solute and solvent is not to change the fluctuations, but to affect the average solvent density and the position of this interface. For strong enough attractions, the effect is to pin the interface at specific positions, as seen in Fig. 4, and to a lesser extent in Fig. 6.

This picture of the role of attractions in the context of hydrophobic solvation has been discussed before $[10,12]$. Simulation results of Werder et al. on the hydration of several different models of graphite in water [24] can be viewed as illustrations of that role. Namely, Werder et al. establish linear trends for hydration energies as functions of solvent-solute attractive energy strength. The relative range of parameters is smaller in that work than in ours, probably explaining why curvature in the linear trends is not apparent. Along with demonstrating regularity and simplicity, Werder et al. also show that the trends are 
significant in that modest potential parameter changes result in models of "graphite" that change from hydrophobic to hydrophilic. In one model, Werder et al. consider a LennardJones potential between graphite carbon atoms and water oxygen atoms with energy and length parameters $\varepsilon_{\mathrm{CO}}=0.39 \mathrm{~kJ} / \mathrm{mol}$ and $\sigma_{\mathrm{CO}}=0.32 \mathrm{~nm}$. They establish that in this model, line tension is positive and the contact angle between water and a planar graphite surface is finite. In contrast, a similar model of a presumed hydrophobic surface, but with $\varepsilon_{\mathrm{CO}}=0.48 \mathrm{~kJ} / \mathrm{mol}$ and $\sigma_{\mathrm{CO}}=0.33 \mathrm{~nm}$ yields a negative surface tension between water and the surface $[25,26]$. This latter surface is therefore not hydrophobic.

An important consequence of the simplicity we emphasize regards the entropy of solvation. To the extent that density fluctuations obey Gaussian statistics (i.e., the mean density responds linearly to forces of adhesion), the entropy of solvation is independent of adhesive energy strength. This idea is used in Ref. 27 to replace the hydration entropy of a protein with that of a hard sphere occupying the same volume, even though the surface of the folded protein is largely hydrophilic. By demonstrating linear trends for the mean density, our work here provides explicit justification for this replacement.

\section{Acknowledgments}

This research has been supported in its early stages by the National Science Foundation and subsequently by U.S. Department of Energy grant No. DE-AC02-05CH11231.

[1] Weeks, J. D. Ann. Rev. Phys. Chem. 2002, 53, 533.

[2] Widom, B. Science 1967, 157, 375.

[3] Weeks, J. D.; Chandler, D.; Andersen, H. C. J. Chem. Phys. 1971, 54, 5237. 
[4] Evans, R. In Liquids at interfaces; Charvolin, J., Joanny, J. F., Zinn-Justin, J., Eds.; NorthHolland, 1988.

[5] Hummer, G.; Garde, S.; Garcia, A. E.; Pohorille, A.; Pratt, L. R. Proc. Natl. Acad. Sci. USA 1996, 93, 8951.

[6] Crooks, G. E.; Chandler, D. Phys. Rev. E 1997, 56, 4217.

[7] Chandler, D. Phys. Rev. E 1993, 48, 2898.

[8] Hansen, J.-P.; Verlet, L. Phys. Rev. 1969, 184, 151.

[9] Smit, B. J. Chem. Phys. 1992, 96, 8639.

[10] Huang, D. M.; Chandler, D. J. Phys. Chem. B 2002, 106, 2047.

[11] Huang, D. M.; Chandler, D. Phys. Rev. E 2000, 61, 1501.

[12] Chandler, D. Nature 2005, 437, 640.

[13] Ashbaugh, H. S.; Paulaitis, M. E. J. Am. Chem. Soc. 2001, 123, 10721.

[14] Mehrotra, P. K.; Beveridge, D. L. J. Am. Chem. Soc. 1980, 102, 4287.

[15] Equivalent to eqs. (14) and (15), the proximal distribution function can be written as $\Omega_{1} r^{2} \rho g_{\text {prox }}(r)=\left\langle\sum_{i=1}^{N} \delta\left(r-\left|\boldsymbol{R}_{i}\right|\right) \prod_{\alpha=2}^{N} \theta\left(\left|\boldsymbol{R}_{i}-\boldsymbol{r}_{\alpha}\right|-\left|\boldsymbol{R}_{i}\right|\right)\right\rangle$. The equilibrium average is performed with solute site 1 at the origin, i.e., $\boldsymbol{r}_{1}=0$, this site being at the surface of the cluster and with subtended solid angle $\Omega_{1}, \delta(x)$ is the Dirac delta function, and $\theta(x)$ is the unit Heaviside function. The quantity $\rho g_{\text {prox }}(r)$ is the mean density of solvent that is closest to and within a shell of radius $r$ from cluster particle $\alpha=1$.

[16] Berendsen, H. J. C.; Postma, J. P. M.; van Gunsteren, W. F.; Hermans, J. In Pullman, B., Ed., Intermolecular forces: Proceedings of the fourteenth Jerusalem symposium on quantum chemistry and biochemistry, page 331, Dordrecht, 1981. Reidel.

[17] Jorgensen, W. L.; Madura, J. D.; Swenson, C. J. J. Am. Chem. Soc. 1984, 106, 6638. 
[18] Plimpton, S. J. J. Comp. Phys. 1995, 117, 1.

[19] LAMMPS - Large-scale Atomic / Molecular Massively Parallel Simulator. Plimpton, S. J. http://lammps.sandia.gov.

[20] Svishchev, I. M.; Kusalik, P. G. J. Chem. Phys. 1993, 99, 3049.

[21] Pratt, L. R.; Chandler, D. J. Chem. Phys. 1977, 67, 3683.

[22] Lum, K.; Chandler, D.; Weeks, J. D. J. Phys. Chem. B 1999, 103, 4570.

[23] Stillinger, F. H. J. Sol. Chem. 1973, 2, 141.

[24] Werder, T.; Walther, J. H.; Jaffe, R. L.; Halicioglu, T.; Koumoutsakos, P. J. Phys. Chem. B 2003, 107, 1345.

[25] Choudhury, H.; Pettitt, B. M. J. Am. Chem. Soc. 2005, 127, 3556.

[26] In Ref. 24, Werder et al. do not report the oil-water surface tension for these particular parameters, but Choudhury and Pettitt, in effect, do so in Ref. 25. Specifically, for carbon-carbon potential parameters, the authors of [25] cite $\varepsilon_{\mathrm{CC}}=0.36 \mathrm{~kJ} / \mathrm{mol}$ and $\sigma_{\mathrm{CC}}=0.34 \mathrm{~nm}$, and combine these with oxygen-oxygen parameters $\varepsilon_{\mathrm{OO}}=0.65 \mathrm{~kJ} / \mathrm{mol}$ and $\sigma_{\mathrm{OO}}=0.32 \mathrm{~nm}$, using geometrical and arithmetic means, respectively, to obtain their carbon-oxygen parameters. They compute a potential of mean force by molecular simulation for a pair of plates formed from these "carbon" particles. Figure. 1a of their paper shows that the solvent contribution to the free energy for dissociated plates is lower than that of associated plates. The plates are reasonably large, so that the solvation energy is predominantly proportional to the solute surface area exposed to the solvent. Choudhury and Pettitt's result for the solvent contribution to the potential of mean force therefore shows that the interactions used in their study produce a negative solvation surface free energy for the hydrated plate.

[27] Huang, D. M.; Chandler, D. Proc. Natl. Acad. Sci. USA 2000, 97, 8324. 
[28] Huang, D. M.; Geissler, P. L.; Chandler, D. J. Phys. Chem. B 2001, 105, 6704. 

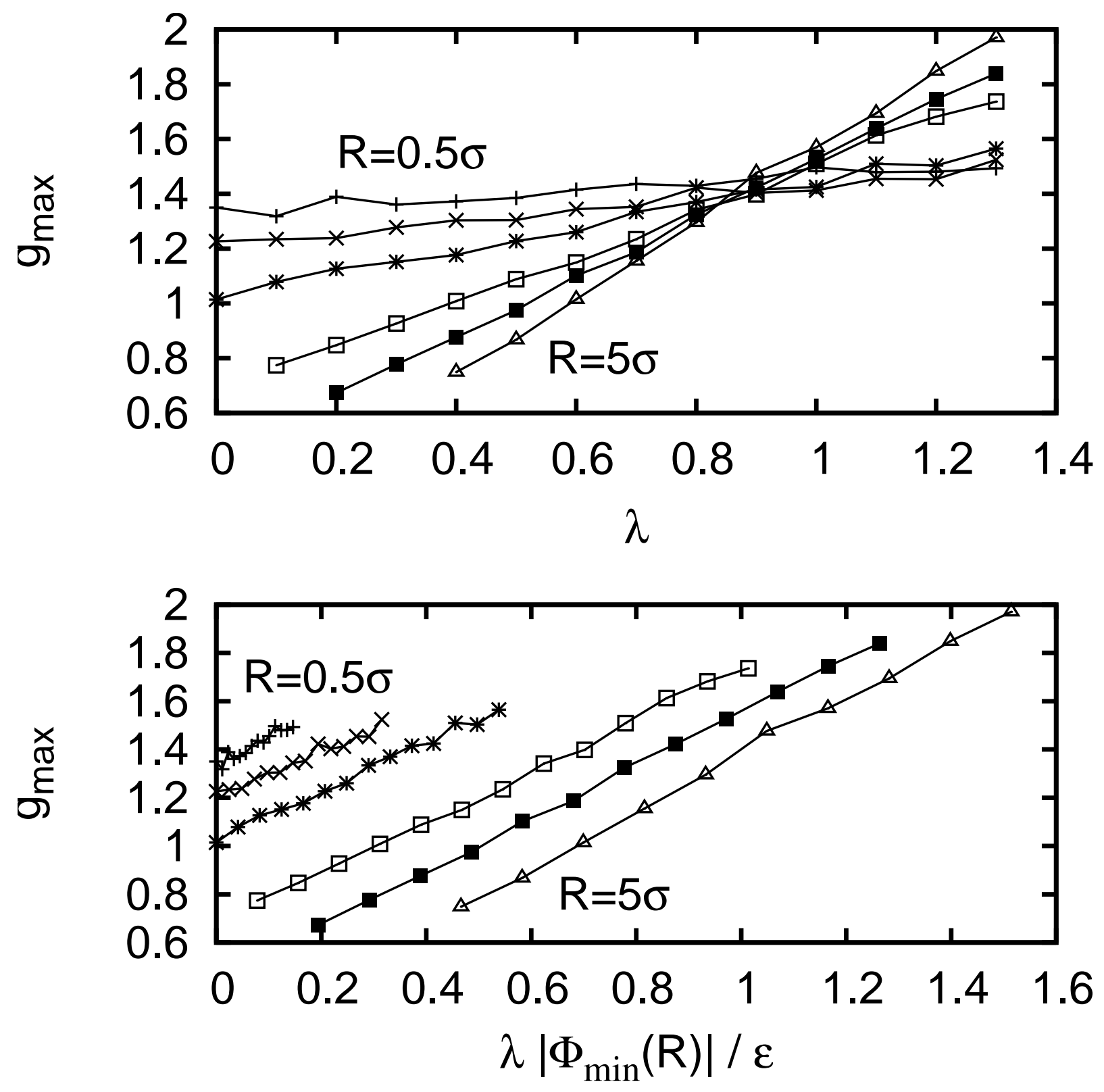

FIG. 1: Magnitude of the first peak of the solvent density distribution function around homogeneous Lennard-Jones solutes of radii $R / \sigma=0.5,0.7,1,2,3,5$, plotted as a function of the bare attraction strength $\lambda$ (top) and the minimum of the solvent-solute potential (bottom). For large solute sizes and small values of $\lambda$ the reduced density distribution function did not have a well-defined first peak, and no data is shown for those systems. 

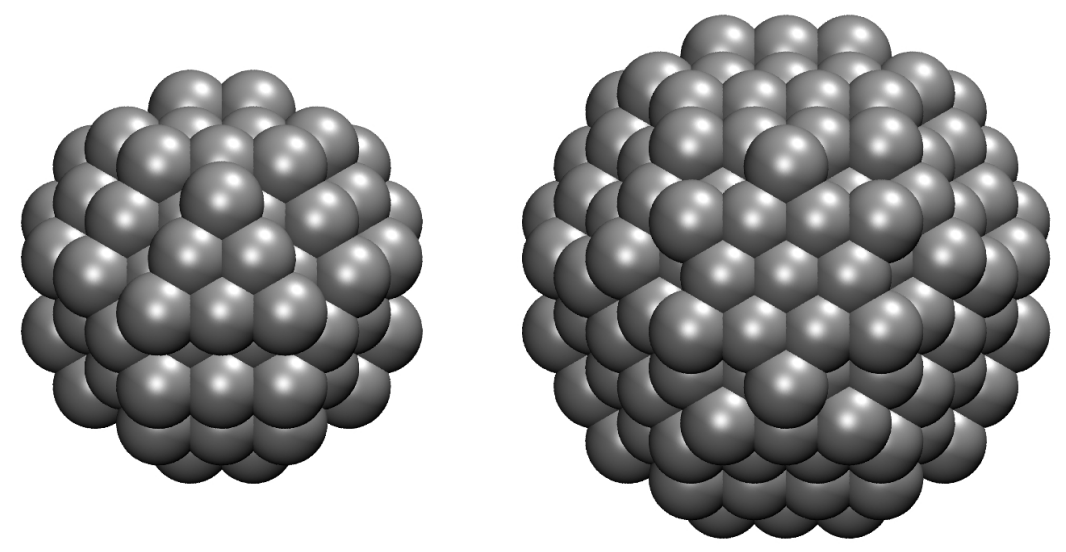

FIG. 2: Space filling view of the solute clusters with particle numbers $n=1,13,57,135,305$. The clusters are spherical cuts of a hexagonally close-packed crystal. 

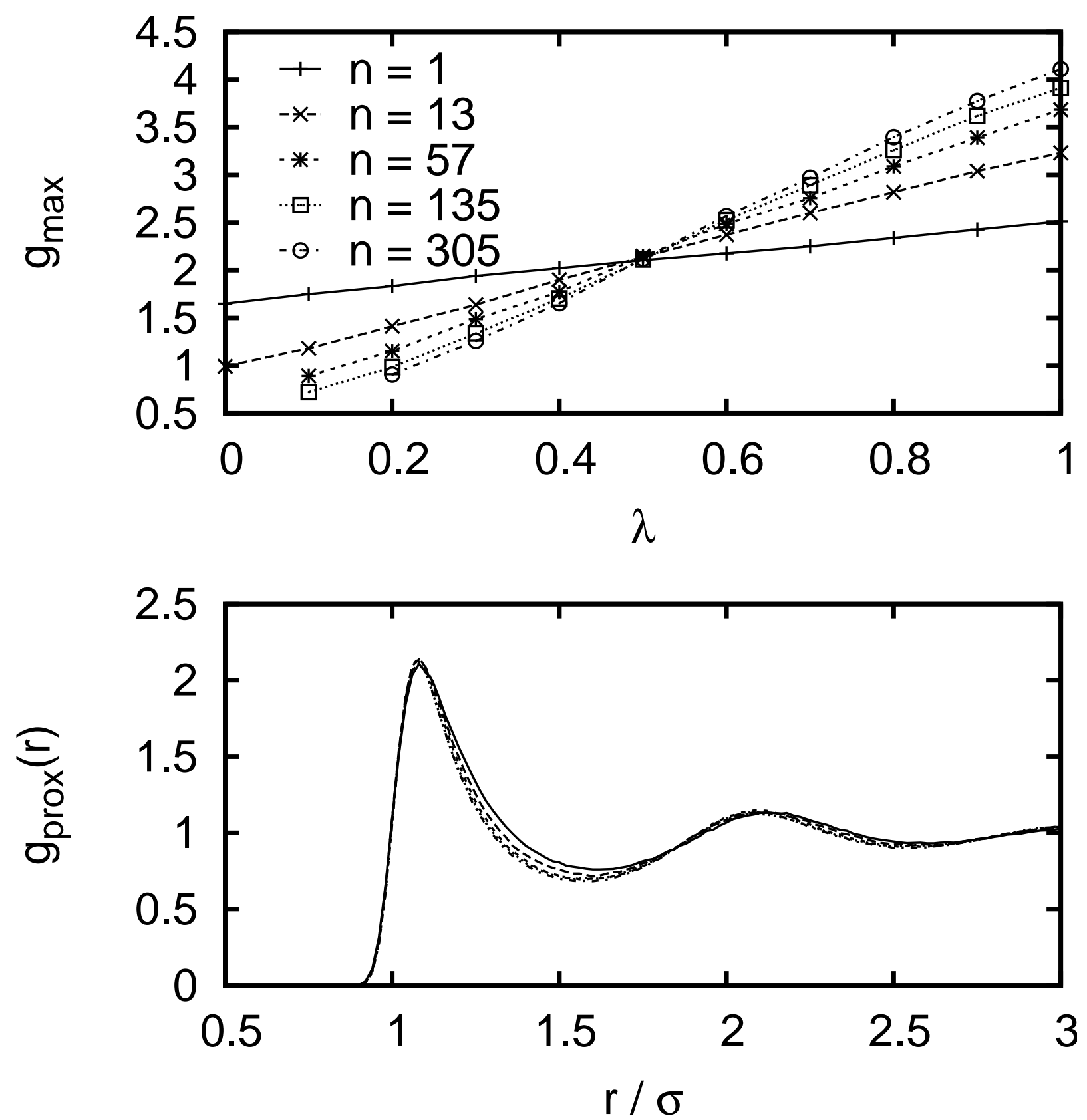

FIG. 3: Proximal solvent density distribution function around clusters of Lennard-Jones particles immersed in the Lennard-Jones fluid. (top) Height of the first peak in $g_{\text {prox }}(r)$ for different cluster sizes $n$ and attraction strengths $\lambda$. (bottom) Proximal distribution functions for $\lambda=\lambda^{*}=0.5$. 


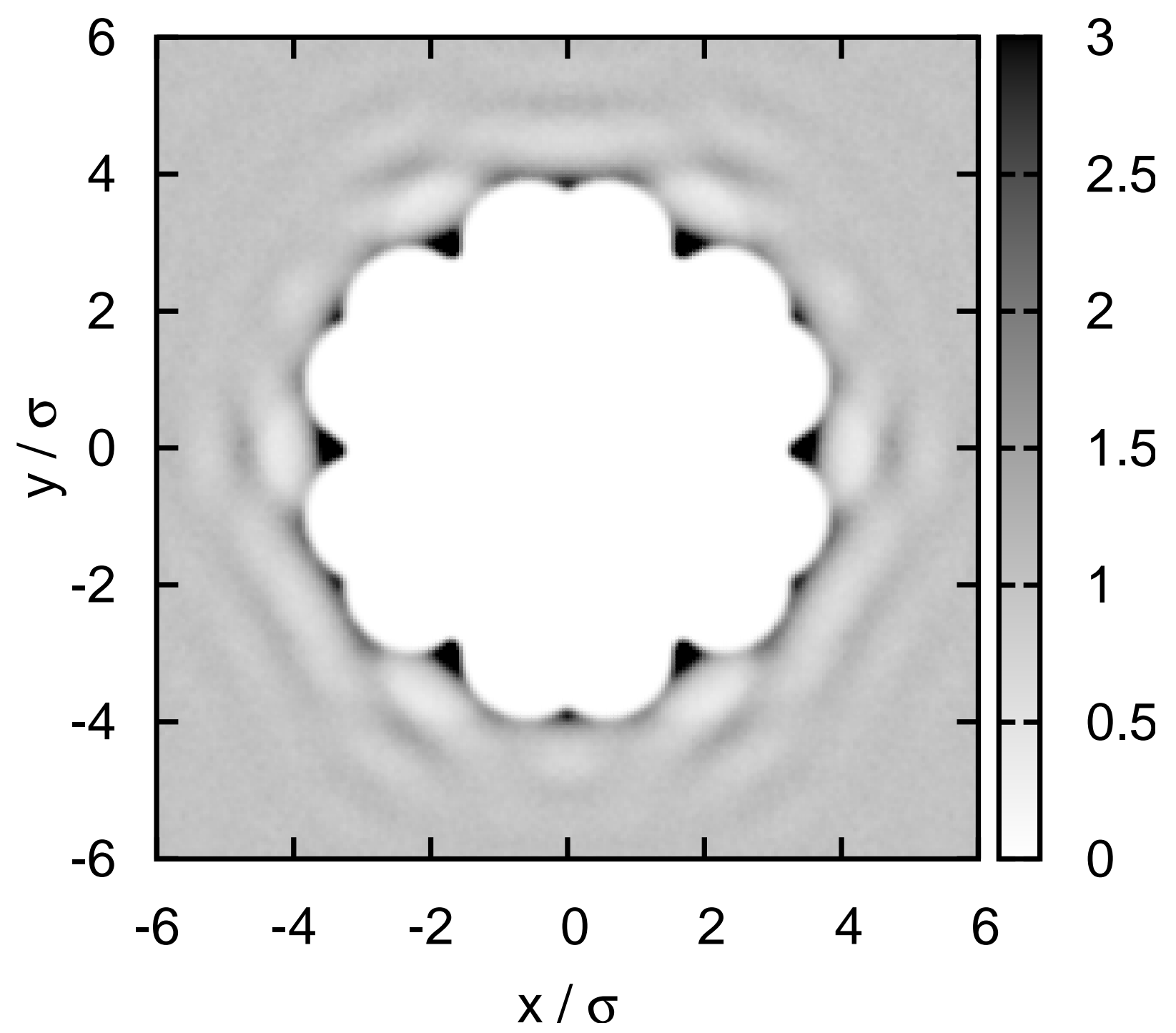

FIG. 4: Reduced solvent density around the $n=135$ solute cluster immersed in the Lennard--Jones fluid at $\lambda=\lambda^{*}=0.5$. Shown is a slice through the solute center of width $0.1 \sigma$ with resolution $0.05 \sigma$, demonstrating that the solvent density does not follow the shape of the solute surface. 


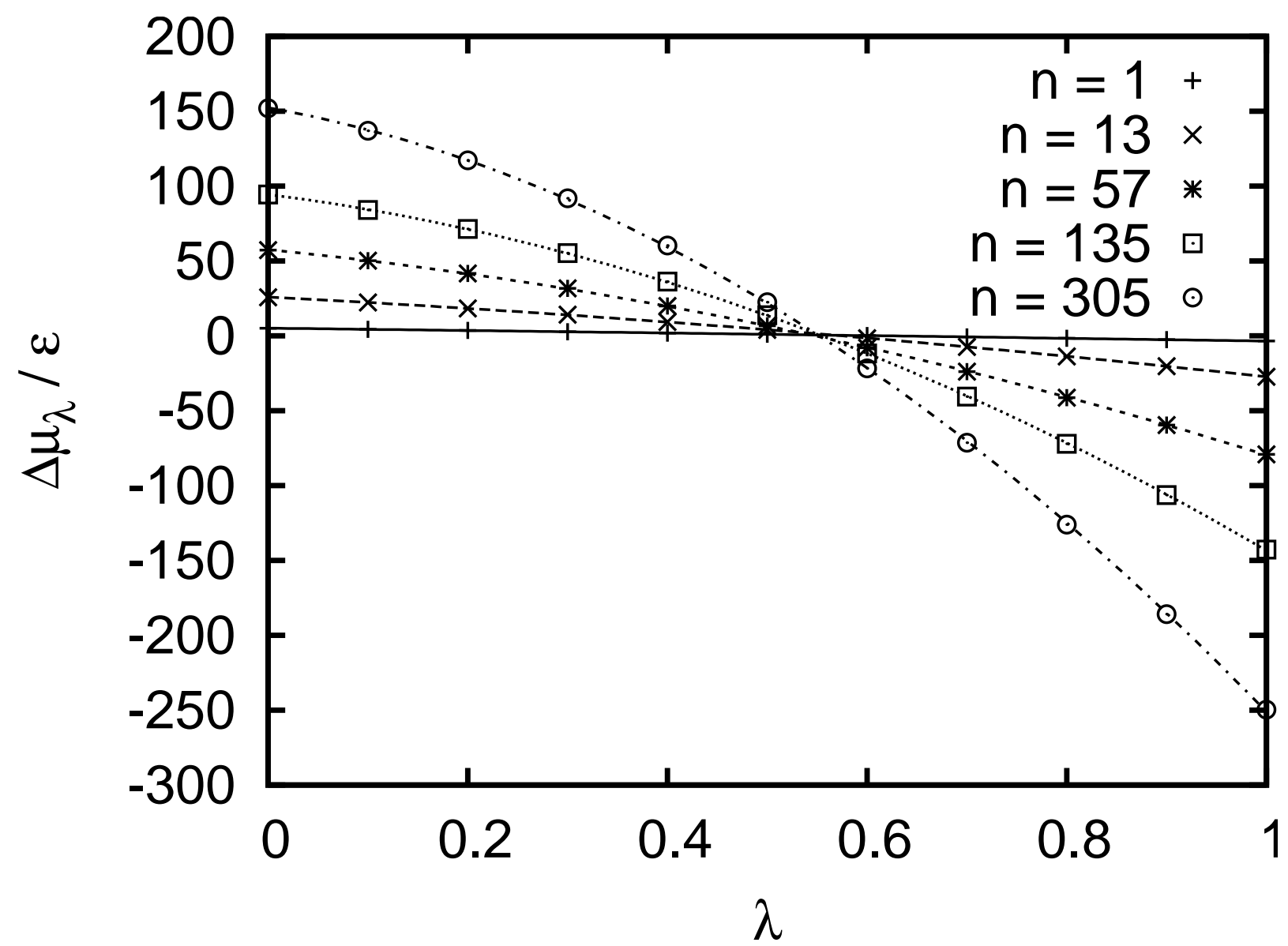

FIG. 5: Excess solvation energy of solute clusters in the Lennard-Jones fluid. The symbols are calculated from simulation using Eq. (16), and the lines are quadratic fits through the data. 


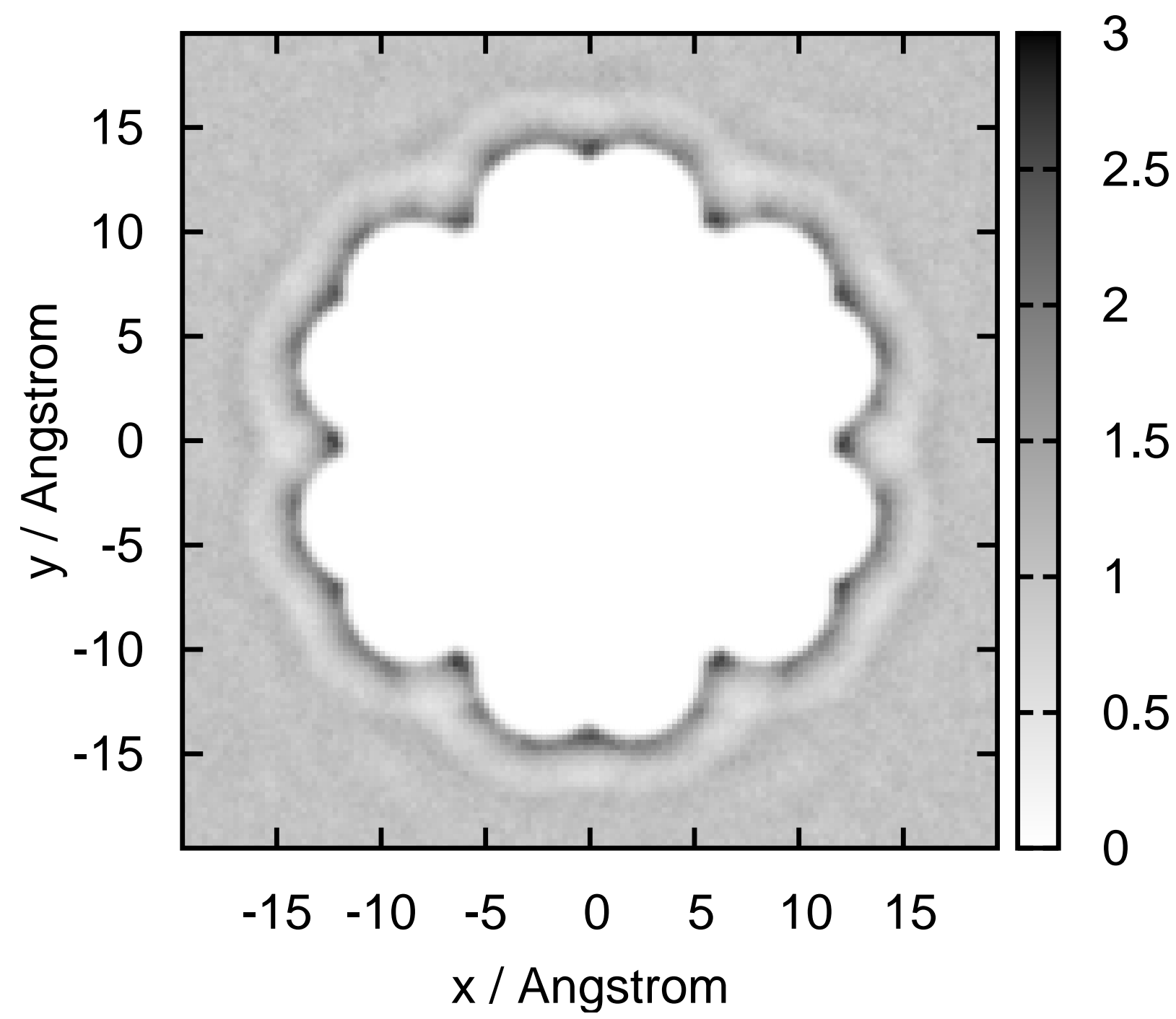

FIG. 6: Reduced water density around the $n=135$ solute cluster at $\lambda=\lambda^{*}=1$. Shown is a slice through the solute center of width $0.5 \AA$ with resolution $0.25 \AA$. To a good approximation the solvent density follows the shape of the solute cluster. 


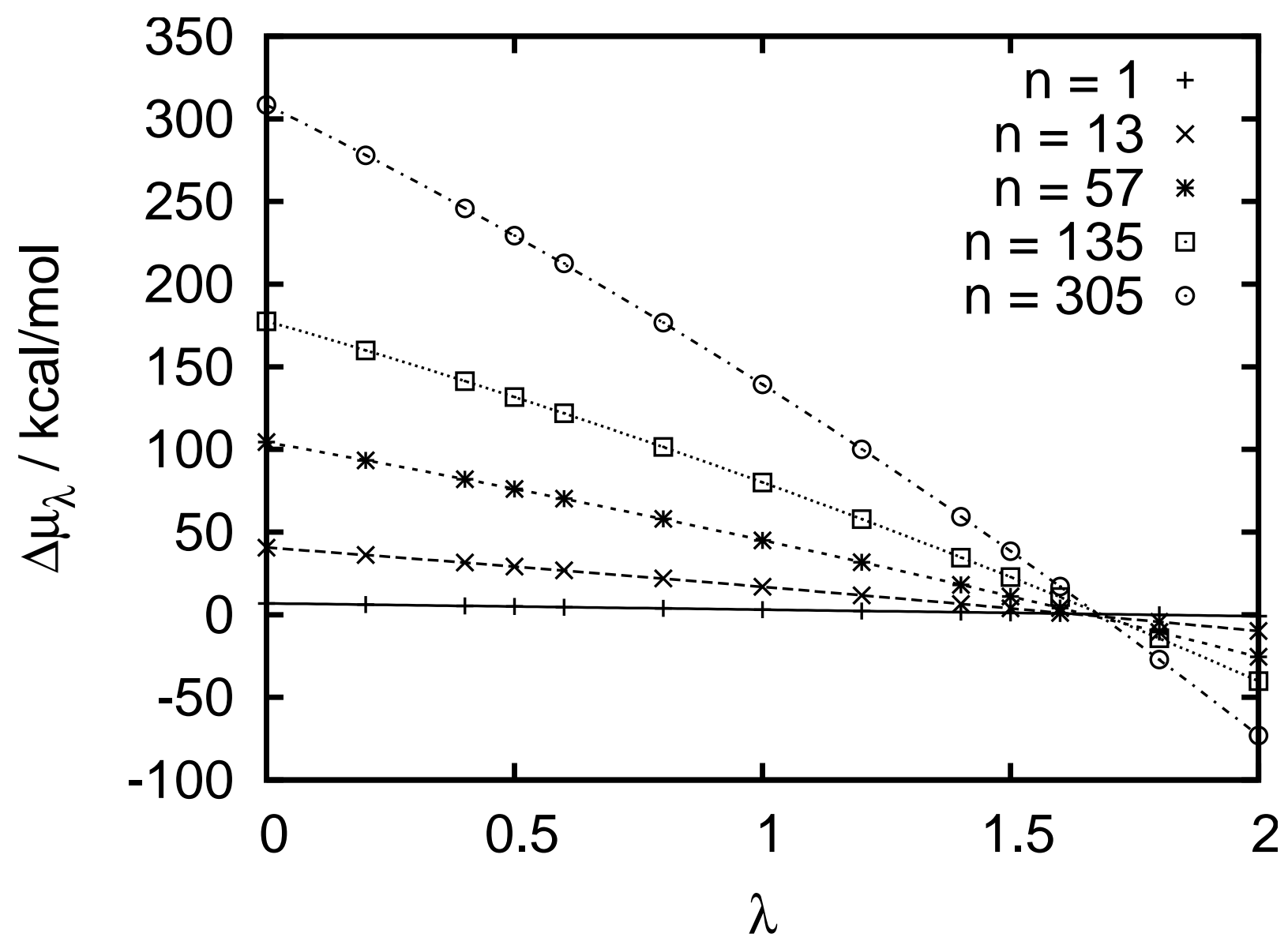

FIG. 7: Excess solvation energy of methane clusters in water. The symbols are computed from simulation with the aid of Eq. (16) and known solvation free energies of hard sphere solutes in water [28]. The lines are quadratic fits to the data. 